\title{
BMJ Open Evaluating the impact of a community health worker programme on non- communicable disease, malnutrition, tuberculosis, family planning and antenatal care in Neno, Malawi: protocol for a stepped-wedge, cluster randomised controlled trial
}

Elizabeth L Dunbar, ${ }^{1,2}$ Emily B Wroe, ${ }^{1}$ Basimenye Nhlema, ${ }^{1}$ Chiyembekezo Kachimanga, ${ }^{1}$ Ravi Gupta, ${ }^{1}$ Celia Taylor, ${ }^{3}$ Annie Michaelis, ${ }^{4}$ Katie Cundale, ${ }^{1}$ Luckson Dullie, ${ }^{1}$ Arnold Jumbe, ${ }^{5}$ Lawrence Nazimera, ${ }^{5}$ Ryan McBain, ${ }^{4}$ Richard J Lilford, ${ }^{3}$ Samuel lan Watson ${ }^{3}$

To cite: Dunbar EL, Wroe EB, Nhlema B, et al. Evaluating the impact of a community health worker programme on non-communicable disease, malnutrition, tuberculosis, family planning and antenatal care in Neno, Malawi: protocol for a steppedwedge, cluster randomised controlled trial. BMJ Open 2018;8:e019473. doi:10.1136/ bmjopen-2017-019473

- Prepublication history and additional material for this paper are available online. To view these files, please visit the journal online (http://dx.doi. org/10.1136/bmjopen-2017018243).

Received 4 September 2017

Revised 2 May 2018

Accepted 14 May 2018

Check for updates

(C) Author(s) (or their employer(s)) 2018. Re-use permitted under CC BY-NC. No commercial re-use. See rights and permissions. Published by BMJ.

For numbered affiliations see end of article.

Correspondence to

Elizabeth L Dunbar;

edunbar@pih.org

\section{ABSTRACT}

Introduction This protocol concerns the implementation and evaluation of an intervention designed to realign the existing cadre of community health workers (CHWs) in Neno district, Malawi to better support the care needs of the clients they serve. The proposed intervention is a 'Household Model' where CHWs will be reassigned to households, rather than to specific patients with HIV and/or tuberculosis (TB).

Methods and analysis Using a stepped-wedge, cluster-randomised design, this study investigates whether high HIV retention rates can be replicated for non-communicable diseases (NCDs), and the model's impact on TB and paediatric malnutrition case finding, as well as the uptake of family planning and antenatal care. Eleven sites (health centres and hospitals) were arranged into six clusters (average cluster population 21800 ). Primary outcomes include retention in care for HIV and chronic NCDs, TB case finding, paediatric malnutrition case finding, and utilisation of early and complete antenatal care. Clinical outcomes are based on routinely collected data from the Ministry of Health's District Health Information System 2 and an OpenMRS electronic medical record supported by Partners In Health. Additionally, semistructured qualitative interviews with various stakeholders will assess community perceptions and context of the Household Model.

Ethics and dissemination Ethics approval has been obtained from the Malawian National Health Science Research Committee (\#16/11/1694) in Lilongwe, Malawi; Partners Healthcare Human Research Committee (\#2017P000548/PHS) in Somerville, Massachusetts; and the Biomedical and Scientific Research Ethics Sub-Committee (REG0-2017-2060) at the University of Warwick in Coventry, UK. Dissemination will include manuscripts for peer-reviewed publication as well as a

\section{Strengths and limitations of this study}

- The stepped-wedge, cluster-randomised controlled trial allows for robust evaluation of a polyvalent community health worker (CHW) programme that requires staggered training and implementation.

- The study will use routinely available data from the Ministry of Health's District Health Information Software II reporting system and an electronic medical record system.

- The trial will estimate the effect of a polyvalent CHW programme on several facility-level health outcomes, but will not be able to isolate specific questions of interest in the programme design, such as CHW household ratios or models of supervision.

- This protocol is not designed to measure the cost-effectiveness of the programme, although an economic evaluation is planned in the future.

full report detailing the findings of the intervention for the Malawian Ministry of Health.

Trial registration number NCT03106727.

Primary sponsor Partners In Health I Abwenzi Pa Za Umoyo P.0. Box 56, Neno, Malawi. Protocol Version 4, March 2018.

\section{INTRODUCTION}

Background and context

In 2007, Partners In Health (PIH), an international non-governmental organisation, was invited to Neno district by Malawi's Ministry of Health (MOH) to strengthen health service delivery. PIH's global mission 
is to create a preferential option for the poor in healthcare by accompanying the public sector in strengthening health services, professional training and mentorship, and targeted research. A central part of PIH's model of care is its community health worker (CHW) programme. Since 2007, PIH, known in Malawi by its Chichewa name Abwenzi Pa Za Umoyo, has relied on a CHW cadre that primarily provides ongoing support and accompaniment to patients with tuberculosis (TB) and HIV attending healthcare facilities. Other PIH-supported sites have demonstrated improved patient adherence to treatment with regular CHW visits, accompaniment to health facilities and social support in the home. ${ }^{12}$ CHWs have also been used to find patients with TB and HIV to reduce the rate default from care.

$\mathrm{MOH}$ and PIH provide HIV care services across all 14 facilities in Neno district, including 2 hospitals and 12 health centres. Neno has achieved some of the strongest HIV outcomes in Malawi, with 12-month survival rates over $10 \%$ points higher than the national average. ${ }^{3}$ The Neno Chronic Care Clinic was established in 2009 at the district hospital to provide ongoing management of non-communicable diseases (NCDs) including hypertension, asthma, epilepsy, diabetes and congestive heart failure. The Chronic Care Clinic expanded to the Lisungwi Community Hospital in 2013 (see figure 1

\section{Neno District Health Facilities}

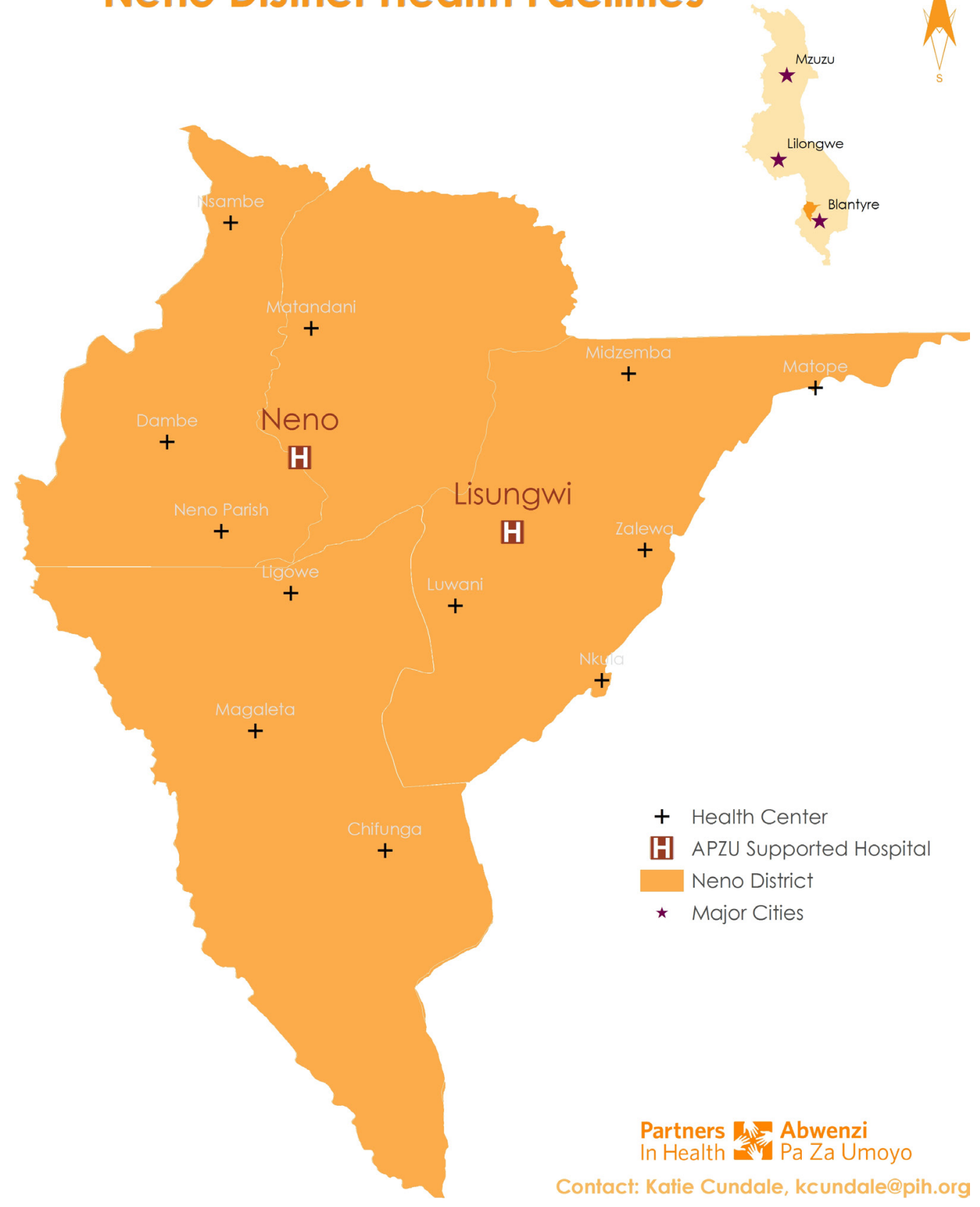

Figure 1 Map of health facilities in Neno district supported by Partners In Health. APZU, Abwenzi Pa Za Umoyo. 
for a map of health facilities in Neno district). In early 2015, the HIV Care Clinic and the Chronic Care Clinic were merged into the Integrated Chronic Care Clinic $\left(\mathrm{IC}^{3}\right)$, which allowed for decentralisation of chronic care to all facilities across Neno. Thus, all clients with chronic conditions, including HIV and NCDs, are managed in $\mathrm{IC}^{3}$ and patients can receive care at the facility closest to their homes. At every IC $^{3}$ visit, all clients undergo routine screening for nutrition, diabetes, hypertension, HIV and TB. However, despite decentralised HIV and NCD care, the CHW programme did not cater to NCD clients and retention in care for NCDs was relatively low.

There are several other health programmes supported in Neno district. In July 2015, PIH also rolled out an integrated screening programme in remote communities: Screening for Health and Referral in the Community (SHARC). Interdisciplinary teams travel from clinics to remote areas of the district two afternoons per week and invite local communities for health education and screening for a variety of conditions including HIV, TB, hypertension, diabetes, malnutrition and family planning. The facility-based twin of this programme, Screening for Health and Referral at the Facility (SHARF), was piloted in 2016 in 3 facilities and in July 2017 was scaled up to 7 of the 14 facilities in the district.

\section{Rationale and design}

The decentralisation of $\mathrm{IC}^{3}$, along with recognition of the role CHWs played in the strong HIV outcomes in Neno district, ${ }^{3}$ provided an opportunity to expand the role of CHWs to focus on the health of entire households rather than the previous focus only on patients already diagnosed with HIV and TB. In the existing model, starting in 2007, CHWs were paired with an average of six HIV and/or TB clients to monitor medication adherence and side effects and accompany clients to clinical visits. This typically involved daily visits to the patients' homes in addition to attending clinic visits. The monthly stipend ranged from US\$24 to US\$28 in 2014-2015. A pilot project in 2015-2016 at two facilities in Neno, where CHWs focused on maternal health in addition to HIV and TB responsibilities, demonstrated some early successes of household-level assignments, including an increase in facility-based deliveries and attendances at antenatal clinics early in pregnancy (Kachimanga et al, 2017, unpublished).

The design of our 'Household Model' was based on existing polyvalent CHW programmes at other PIH sites and on the burden of disease prioritised by the $\mathrm{MOH}$. The team reviewed the existing curricula, data collection mechanisms and CHW tracking, as well as considered key questions in Developing and Strengthening Community Health Worker Programmes at Scale: A Reference Guide for Programme Managers and Policy Makers. ${ }^{4}$ In addition, in partnership with the clinical team, the CHW programme considered the major burdens of disease in Malawi that were conducive to a CHW intervention, as well as the clinical priorities being addressed by the district $\mathrm{MOH}$ leadership, including maternal-child health, HIV, NCDs and TB. These priorities informed the components of the Household Model, as did the general shift towards integrated care at the health facility level described above.

In the new Household Model, each CHW will be responsible for visiting around 20-40 households once every month, with more frequent visits to households with members enrolled in chronic clinical care. During these home visits, CHWs are responsible for case finding through education and screening for common conditions, including STIs, TB, HIV and paediatric malnutrition. They will provide support for linkage to care for symptomatic clients or women who may be pregnant, along with ongoing support and accompaniment for patients in care, including adherence support, psychosocial support and tracking of missed patient visits.

The objectives of this study are to estimate the effect of the CHW Household Model-as compared with the pre-existing HIV/TB-specific CHW model-on:

- Retention in care for patients with chronic NCDs, specifically hypertension, asthma, diabetes, epilepsy and mental health.

- Case finding and enrolment into the MOH's Childhood Management for Acute Malnutrition programme for children under 5 years with malnutrition.

- Retention in care for patients living with HIV.

- Case finding and enrolment into the MOH's TB programme for TB treatment.

- Uptake of women's health services including family planning (particularly long-term methods), as well as early and complete antenatal care.

\section{Filling the evidence gap}

There is evidence throughout the literature on the benefit of CHWs at the household level in terms of disease prevention, increasing adherence and access to care, encouraging behavioural change, and on specific outcomes such as decreased neonatal, child and maternal mortality (see, eg, Calcagno et al, 2016; Lewin et al, 2010; McCord et al, 2015; Pallas et al, 2013; Perry et al, 2014; Singh et al, 2015).

As with other areas of public health and healthcare, there has been a drive towards more comprehensive, integrated approaches to healthcare coverage enacted through CHWs as opposed to the vertical, disease-specific nature of some CHW programmes. ${ }^{5}{ }^{6}$ Disease-specific programmes can sometimes have detrimental effects on health systems because of their focus on single disease burdens. ${ }^{7}$ While there is growing evidence of the benefit of using CHWs to provide more comprehensive care, ${ }^{68}$ there is also a frequently cited lack of research into which conditions or tasks should be integrated into CHW roles. ${ }^{9-11}$ The need to provide high-quality evidence on the expansion of CHW roles is crucial, as studies have found that the broadening of roles or responsibilities has led to CHWs not providing the full spectrum of expected care ${ }^{12}$ CHWs feeling stressed due to added expectations from community members ${ }^{13}$ or because they felt they were 
responsible for more than they could handle. ${ }^{11}$ Further research into the optimal 'dose' of tasks for which CHWs are responsible will provide evidence regarding how best to organise integrated, comprehensive care.

We therefore hope to contribute to the literature by providing robust evidence of the effects of an integrated CHW programme on a wide range of outcomes. Given the large amount of evidence on HIV and other single disease CHW programmes, we hope to provide new insights into whether broadening services offered by CHWs can influence health outcomes.

\section{METHODS AND ANALYSIS \\ Study location}

Neno district is a rural and impoverished district in southwest Malawi, with an estimated population of approximately 165000 in $2017 .{ }^{14}$ Located along the border of Mozambique in a remote and mountainous region, Neno District Hospital is not accessible by tarmac roads. The majority of the population is subsistence farmers and $95 \%$ of households are without electricity. ${ }^{15}$ The socioeconomic status of Malawi is poor in general, where nationally the average income is US $\$ 1$ per day. ${ }^{16} \mathrm{HIV}$ prevalence in Malawi's south-west zone was as high as $15 \%$ in $2010,{ }^{15}$ the highest in the country; 2014 national statistics indicate a $10 \%$ average national prevalence. ${ }^{17}$ Malawi was listed by WHO as one of the 30 countries with high TB/HIV burden, ${ }^{18}$ and malnutrition rates (prevalence) in children under 5 years are around $4 \% .{ }^{19}$ Maternal mortality in the country is also the highest in the world at 439 per 100000 live births, and Neno district data show that only $57 \%$ of married women are on modern family planning. ${ }^{20}$ NCD care, while listed in the Essential Health Package in Malawi's Health Sector Strategic Plan II, is extremely limited in the rural areas, and population data suggest that up to a third of Malawian adults suffer from hypertension and over $5 \%$ from diabetes. ${ }^{21}$

\section{Study design: stepped-wedge, cluster-randomised trial}

The implementation of the new CHW model is designed so that it may be evaluated as a stepped-wedge, cluster-randomised trial (SW-CRT) (figure 2). The steppedwedge study design was selected for a number of reasons. First, the training of CHWs needs to be staggered due to training capacity constraints. Second, all sites in Neno will receive the intervention. And third, the stepped-wedge RCT design permits estimation of the causal effects of the intervention. ${ }^{22}{ }^{23}$ We plan to conduct a long-term follow-up at 3 years postimplementation making use of routinely collected data to determine whether any effects observed in the primary analysis persist over time. Additionally, we plan to conduct an economic evaluation of the programme in the future.

\section{Sample selection}

Eleven intervention sites were clustered into six groups based on population size such that each group had a manageable number of CHWs to train. The order of implementation for these six sites was randomised by a third party. In the SW-CRT study design, each cluster crosses over from the control group to intervention group until all groups have received the intervention. The six clusters were created from the 11 sites based mostly on geographical location and on catchment area sizes (mean population 21848 , range 12515 to 27426 ). There are no exclusion criteria, as the study aims to assess whether CHWs assigned to more households and with a more integrated care system (rather than just HIV and TB), will be able to more effectively reach households in need of health support. Households can refuse the CHW's service; however, based on our 10 years experience with CHWs working in Neno, this is a rare occurrence.

\section{Recruitment, hiring and supervision of CHWs}

Each CHW will serve an average of 30 households (range 20-40). Household is defined by the family unit and the head of household, encompassing all of the dependents that individual is providing for. This ratio was chosen accounting for household spacing and difficult terrain in Neno; the anticipated CHW workloads including monthly home visits and longitudinal support for approximately 12 patients with chronic illness and six pregnant women; expert advice from CHWs and CHW programme leaders; and the need to have CHWs in every village living in the communities they serve. In addition, a previous pilot study tested a ratio of one CHW to 20 households, and this study will use a higher ratio in order to test a less costly model. The ratio will vary slightly based on the geography and household spacing of given catchment areas. Each of Neno's 138 villages will be assigned enough CHWs to meet this requirement. The CHW cadre in

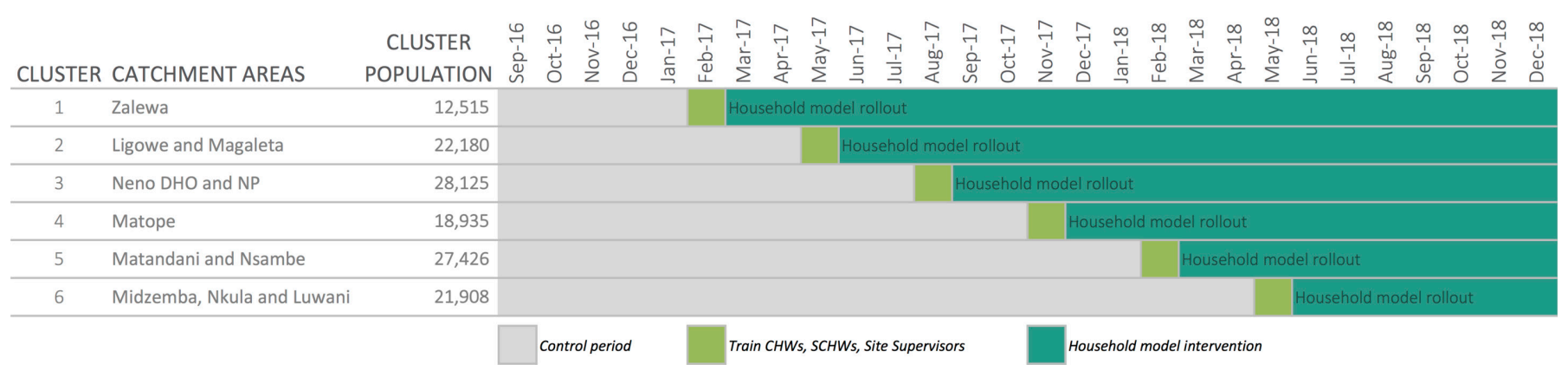

Figure 2 Household model stepped-wedge roll-out. CHW, community health worker; SCHW, senior community health worker. 
Neno will grow from 1000 CHWs to approximately 1200. Each CHW will receive a monthly stipend of Malawian Kwacha (MWK) 15000 or approximately US\$21. Additionally, all CHWs will be supervised at the village level, with one senior CHW (SCHW) assigned per village. For larger villages ( $>300$ households), two or more SCHWs will be assigned. If the villages are small and proximate, one SCHW will oversee multiple villages. SCHWs will also be responsible for 15 households in addition to their supervision duties. After rolling out the Household Model, we estimate 185 SCHWs will be assigned. SCHWs will be paid a monthly stipend of MWK23000 or approximately US $\$ 32$.

Each community will be briefed about the Household Model programme and the CHW management team will call village heads and local community structures together to discuss the criteria for hiring CHWs and the number of new CHW hires needed in the area. The village heads and community structures will nominate twice as many candidates as the number required.

The CHW selection criteria are:

- Personal requirements:

- Acceptance: approval by the community in which they will work.

- Time: ability to perform tasks without sacrifice of other commitments, with an average commitment of 20 hours per week.

- Literacy: ability to read data forms and educational resources.

- Influence: ability to motivate others.

- Communication skills: ability to effectively communicate pertinent health education messages to all household members.

- Good health: ability to travel long distances on foot.

- Compassion: ability to empathise, relate and support household members.

- Programme requirements:

- A ratio of households to CHWs that is feasible given the number of household members, patients in the catchment area and expected accompaniments required in the health facility.

- Supervision and mentorship close to the community to ensure CHW performance.

Following nomination, candidates will be invited to take a written exam to assess reading and writing skills, as well as basic health knowledge. The top candidates who pass this exam are invited to attend the CHW training.

SCHWs will be selected based on the following criteria:

- Typically, they are previously employed as a CHW.

- Living within the same village or community where they will work.

- Mature, respected and have a history of confidentiality.

- Demonstrated leadership and/or problem-solving skills during the CHW training.

- Ability to influence others.

- Demonstrated above-average understanding of the curriculum.

- Strong data aggregation skills.
- Should be someone that the other CHWs will respect and work well with.

- Should be flexible and adaptable to changes.

- Preference will be given to those with stronger English skills after meeting the above criteria.

\section{Training CHWs}

All CHWs in Neno will be reassigned and trained in the Household Model in a staggered roll-out over 2 years. The maximum number of trainees per group is capped at 60 participants, with some trainings occurring with two groups of CHWs per catchment area.

CHWs will receive a 5-day foundational training. The purpose of the foundational training is to orient CHWs to the following: the new programme and its objectives; their cross-cutting roles; their specific tasks for each primary health focal area; the data collection and documentation requirements; and the knowledge, skills and attitudes needed to successfully perform the aforementioned tasks. The training is structured around the core home visits, which is the key point of interaction between CHWs and their clients.

\section{Evaluation of CHWs}

Along with a rigorous evaluation plan for the recruitment of CHWs and SCHWs, trainings and daily performance of the CHWs will be evaluated throughout the roll-out and continued existence of the Household Model.

CHWs will be evaluated on health knowledge through pretests and post-tests at each training session. A pass mark for each test will be set using the Ebel method of standard setting. A panel of 8-12 PIH and MOH leaders will be established for this process, with these experts required to have a good knowledge of the Household Model, as well as an understanding of the knowledge a CHW needs to be successful in his or her role. The group will collaboratively agree on a definition of the knowledge expected of a 'minimally competent' CHW which will be used as the reference point for determining the pass mark. Furthermore, CHWs will be continuously evaluated based on training attendance, as well as specific knowledge and skills.

Supervision meetings will be held regularly to ensure timely communication between different levels of staff is maintained. The following supervision meetings are core to the Household Model: CHW management meetings; site supervisor meetings; SCHW meetings; general meetings; and village-level CHW meetings. Additionally, SCHWs and site supervisors will conduct monthly supervision home visits, and monthly spot checks will be performed in order to: assess the performance of CHWs and SCHWs; identify areas of support; and identify gaps where the site supervisor or SCHW mentor can provide more clarity or support to CHWs.

\section{Community sensitisation and mobilisation}

PIH's dedicated community education and sensitisation team, the Community Programme (CP) team, will 
be responsible for working with the CHW and clinical teams to keep the community leaders, stakeholders and community members informed and updated about the Household Model. This will encompass education about the role of CHWs in the community, specifically as they shift from an HIV/TB focus to broader household health. These educational sessions will also include the objectives and aims of the model, the timeline, and any changes necessary as the CHW and clinical teams make adjustments along the way. CP will also be a key recipient of information from the community and its leaders, be it challenges, questions for clarification, concerns or successes.

\section{Patient and public involvement}

The development of the Household Model design was rooted in the needs of the population in Neno, based around: the burden of disease; health outcomes data; and feedback from existing patient recipients of the CHW programme. The original shift to household assignments, piloted in two catchment areas, was co-designed by clinical and CP staff in consultation with community members, patients with HIV and community leaders in order to tackle maternal health issues. Focus group interviews with these stakeholders were held to refine the programme details. This was piloted over 18 months, with lessons learnt incorporated into the design of the Household Model.

Patients were involved as community members in the focus groups in the original design, with community forums and community leaders consulted in the programme expansion into the eventual Household Model.

The launch of the Household Model was conducted through multiple forums including the District Executive Council, the health facility management staff, and in public forums through community leaders. The eventual results of this trial will be distributed in the same manner.

The burden of the intervention for the randomisation was not assessed by patients, as this is a cluster randomised trial, with randomisation at health facility level, not by patient level. All clusters will eventually receive the intervention. Stepwise scale-up was essential because of logistical and training needs, so the order of implementation was randomised.

\section{Outcomes to be measured}

Tables 1 and 2 describe the primary and secondary outcomes to be measured at the cluster level by this study.

$\mathrm{MOH}$ data for family planning, malnutrition, TB, antenatal care and maternity are already collected from every health centre and hospital through monthly, standardised reports. These reports summarise aggregated data from standard MOH registers. Registers are populated with information captured from individual patients' health passports, where clinical providers document health details from their facility visit including diagnoses, prescriptions and follow-up instructions. Reports are

\begin{tabular}{|c|c|}
\hline Condition & Measure \\
\hline $\begin{array}{l}\text { Retention in HIV } \\
\text { care program }\end{array}$ & $\begin{array}{l}\text { Proportion of clients taking anteretrovirals } \\
\text { who default from care this month }\end{array}$ \\
\hline $\begin{array}{l}\text { NCDs retention } \\
\text { in care }\end{array}$ & $\begin{array}{l}\text { Proportion of NCD clients who default from } \\
\text { care this month }\end{array}$ \\
\hline $\begin{array}{l}\text { Paediatric } \\
\text { malnutrition }\end{array}$ & $\begin{array}{l}\text { Proportion of children aged } 6-59 \text { months } \\
\text { newly enrolled in care for moderate or } \\
\text { severe paediatric malnutrition }\end{array}$ \\
\hline Tuberculosis & $\begin{array}{l}\text { Proportion of the total population } \\
\text { diagnosed with newly confirmed TB cases }\end{array}$ \\
\hline \multicolumn{2}{|l|}{ Women's health } \\
\hline $\begin{array}{l}\text { Family } \\
\text { planning }\end{array}$ & $\begin{array}{l}\text { Proportion of women of childbearing age } \\
\text { newly starting family planning methods }\end{array}$ \\
\hline $\begin{array}{l}\text { Antenatal } \\
\text { care (ANC) }\end{array}$ & $\begin{array}{l}\text { Proportion of women starting ANC within } \\
\text { the first trimester }\end{array}$ \\
\hline
\end{tabular}

NCD, non-communicable disease; TB, tuberculosis.

entered for each facility into the MOH District Health Information Software II (DHIS2). All data are available on a monthly basis, except for TB data which are captured during quarterly supervisory visits.

The PIH electronic medical record (EMR) captures patient-level data for clients enrolled in HIV and/or NCD care, which includes hypertension, asthma, diabetes, epilepsy and mental health. Data are captured from standardised MOH master cards for each patient. Aggregate data by health facility from the EMR are produced in automated reports generated by the PIH staff.

For each health facility, data on the outcomes will be extracted from the DHIS2 and EMR for the period from October 2016 to July 2019.

To our knowledge, there are currently no other ongoing interventions or planned interventions that we expect will occur at the district level that would systematically compromise our results. PIH is the only non-governmental organization (NGO) working with the $\mathrm{MOH}$ across all district health centres and supporting the $\mathrm{MOH}$ in all of the disease focus areas described here. There are currently plans for one NGO to implement volunteer-based community sputum collection points across five catchment areas that is funded by the Global Fund. Additionally, in three non-study catchment areas along with one Household Model intervention site, the PIH plans to implement a 2-year randomised controlled trial to test models that promote household usage of treatment for potable water. This intervention will not address any conditions to be measured in the Household Model; however, it risks task oversaturation for CHWs involved in the water treatment study. Over time, we will track any new interventions and we will also measure unexpected influences like natural disasters, national stock-outs, or fuel shortages during the intervention that may impact our results.

Additionally, we will track key programme changes that may affect the primary outcomes. We expect an overall 


\begin{tabular}{ll} 
Table 2 Secondary outcomes \\
\hline Condition & Measure \\
\hline HIV programme & $\begin{array}{l}\text { Proportion of ART clients who were } \\
\text { retained in care and surviving } \\
\text { Proportion of population tested for } \\
\text { HIV }\end{array}$
\end{tabular}

NCDs retention in care

NCD programme Proportion of NCD clients who were outcome retained in care and surviving

Hypertension retention Proportion of hypertension clients in care who default from care this month

$\begin{array}{ll}\begin{array}{l}\text { Asthma retention in } \\ \text { care }\end{array} & \begin{array}{l}\text { Proportion of asthma clients who } \\ \text { default from care this month }\end{array} \\ \text { care } & \begin{array}{l}\text { Proportion of diabetes clients who } \\ \text { default from care this month }\end{array} \\ \text { care } & \begin{array}{l}\text { Proportion of epilepsy clients who } \\ \text { default from care this month }\end{array} \\ \text { Mental health retention } & \begin{array}{l}\text { Proportion of mental health clients } \\ \text { in care }\end{array} \\ \text { Malnutrition } & \begin{array}{l}\text { Proportion of children aged } \\ \text { 6-59months who were discharged } \\ \text { as cured in treatment programs for } \\ \text { moderate or severe malnutrition (cure } \\ \text { rate) }\end{array} \\ \text { Moderate malnutrition } & \begin{array}{l}\text { Proportion of children aged } \\ 6-59 \text { months newly enrolled in }\end{array} \\ & \begin{array}{l}\text { the moderate paediatric malnutrition } \\ \text { programme }\end{array} \\ & \begin{array}{l}\text { Proportion of children aged } \\ 6-59 \text { months newly enrolled in } \\ \text { the severe paediatric malnutrition } \\ \text { programme }\end{array} \\ \text { Severe malnutrition } & \end{array}$

Paediatric malnutrition Proportion of children aged inward enrolment $\quad 6-59$ months enrolled in the nutritional rehabilitation unit (inward)

$\begin{array}{ll}\text { Tuberculosis } & \begin{array}{l}\text { Proportion of tuberculosis cases } \\ \text { completing treatment successfully } \\ \text { (no loss to follow-up or death) }\end{array}\end{array}$

Women's health

\begin{tabular}{cl} 
Family planning & $\begin{array}{l}\text { Proportion of women of childbearing } \\
\text { age receiving long-term family } \\
\text { planning methods }\end{array}$ \\
Antenatal care & $\begin{array}{l}\text { Proportion expected pregnant } \\
\text { women in ANC } \\
\text { Proportion of number of women in } \\
\text { the cohort attending more than four } \\
\text { ANC visits }\end{array}$ \\
CHW retention & Proportion of CHWs retained \\
\hline
\end{tabular}

$\mathrm{CHW}$, community health worker; NCD, non-communicable disease.

increase in enrolment given the plans to strengthen and add routine, integrated screening at outpatient departments (SHARF) and continued screening in the community (SHARC). Thus, as this CHW Household Model intervention is rolled out, the size of the cohorts with chronic conditions-namely HIV, TB, hypertension and diabetes-is anticipated to concurrently increase. Furthermore, routine health activities in the district will continue, such as periodical mass screening campaigns for nutritional disorders. There are some parts of the district where CHWs have previously been instrumental in these periodic campaigns, therefore we will continue to document these efforts so that they can be considered in the analysis. Though nutrition is the most extreme example, CHWs have been long-standing members of the healthcare team in Neno, periodically working with $\mathrm{MOH}$ employees to encourage family planning, antenatal care, HIV testing and TB screening, though never in a systematic, structured or longitudinal way. Additionally, the study staff will keep track of other forces that often influence trends in a health system without significant resiliency. For instance, over the past few years Neno district has seen floods, drought, famine, fires and a typhoid epidemic, with corresponding health system responses.

\section{Household qualitative assessment}

In addition to the quantitative data collection described above, we will conduct short semistructured qualitative interviews with a purposively selected sample of around 40 CHW programme recipients. The interviews will be undertaken in November 2017, aiming for a group of respondents with diversity in key characteristics, including socioeconomic status, household location, sex of the household head and length of participation in the programme (eg, families who started receiving CHW support before the roll-out of the Household Model, and families who first received CHW support more recently). Interviews will be conducted until saturation has been reached: in other words, new interview participants will be sought within each cluster until the interview responses are producing no new major themes or ideas. The online supplementary file provides the draft interview guide and informed consent script to be used during recruitment. Transcripts will be analysed for emerging themes using grounded-theory methods, and a codebook will be created to assist with data analysis. Depending on these findings, the team may undertake repeat interviews (with different participants) at the end of the roll-out to determine consistency across catchment areas.

Data will be collected using field workers familiar with the study area. All raw data will be kept in a locked cupboard of a locked office, and all transcribed and translated data will be kept in password-protected files.

\section{Data analysis plan}

Data will be extracted from the DHIS2 and EMR records at the cluster level. Our analysis will follow standard guidelines for the analysis of stepped-wedge, culster randomized control trials- ${ }^{22}$ which specify a model at the cluster level for each time period. The implementation order is randomised, and the model captures the difference between clusters and trends over time, which allows us to estimate the treatment effect of the intervention. Each cluster contains between one and three health 
facilities. As such, we specify a model for the cluster-time cell means. Given the outcome is restricted to lie between 0 and 1 , we specify a generalised linear model. We will consider different link functions: probit, logit, linear and a $\beta$-regression model. To select among these link functions, we will use the Watanabe-Akaike Information Criterion and graphical posterior predictive mode checks, comparing the observed data to samples from the posterior predictive distribution. To allow for extreme 0 and 1 observations in the $\beta$-regression model we will use the transformation $y_{i t}(n-1)+0.5 / n$.

The model for the primary analysis, for cluster $j$ at time $t$ is

$$
\begin{aligned}
\operatorname{Pr}\left(y_{j t} \mid D_{j t}\right) & =f\left(D_{j t} \delta+\alpha_{j}+\theta_{t}\right) \\
\alpha_{j} & \sim N\left(0, \sigma_{\alpha}^{2}\right)
\end{aligned}
$$

Where $y_{j t}$ is the outcome, $D_{j t}$ an indicator for whether cluster $j$ had the intervention at time $t, \theta_{t}$ is the time fixed effect, $\alpha_{j}$ is a normally-distributed cluster random effect with variance $\backslash$ sigma ${ }^{\wedge} 2$ _lalpha and $f($.$) is a link function.$ The model parameters will be estimated for all outcomes. An expert elicitation workshop was conducted to quantify beliefs about treatment effects on the primary outcomes. The elicited distributions will serve as both the prior distribution for the treatment effect parameters and in the power calculation (see below). Weakly informative prior distributions will be specified for the other variables on the basis of previous studies. ${ }^{24}$ Standard deviations will be assigned a half-t(4) distribution. The remaining parameters will be assigned $N\left(0,5^{2}\right)$ priors.

Sensitivity analyses will be conducted with respect to the choices of prior distribution. An uninformative $\mathrm{N}\left(0,10^{2}\right)$ prior distribution will be used for the treatment effect, and half-normal $(0,3)$ distributions for the within-cluster and between-cluster variance.

\section{Power calculation}

We conducted a Bayesian assurance analysis-analogous to a frequentist power calculation. ${ }^{25}{ }^{26}$ We determined the probability that there will be at least a $95 \%$ posterior probability that the treatment effect parameter is $>0$ (or $<0$, where appropriate). We conducted a series of simulations ${ }^{27}$ of trial data to simulate observations from the posterior distribution of the treatment effect parameter. The assurance is calculated on the basis of a design and analysis stage: ${ }^{25}{ }^{26}$ for the design stage we assumed weakly informative $N\left(0,5^{2}\right)$ priors on the treatment effect parameter and we simulated data using parameter distributions for the primary outcomes elicited from experts. For all the outcomes, except HIV/ART retention in care, there was $>95 \%$ probability that there will be at least $95 \%$ posterior probability that the treatment effect parameter is greater than zero. Only a small negative change was expected in HIV/ART retention in care, we estimated that there is a $75 \%$ probability that there will be at least $95 \%$ posterior probability that the treatment effect parameter is less than zero.

\section{ETHICS AND DISSEMINATION}

For the quantitative analyses the data are routinely collected for service evaluation and will be aggregated at the health facility level by month, except for TB data which will be aggregated at the health facility level each quarter. As such, no patients will be identifiable from any quantitative data collected in the study. The National Health Science Research Committee of Malawi was content that informed consent is not necessary for these data. For the qualitative study, informed consent for each interview and its recording will be sought prior to the interview process. The informed consent form is provided in the online supplementary file 1.

The confidentially of all participants in the study, including CHWs, community members, health centre staff and PIH staff, will be maintained. All qualitative data will be anonymised to researchers with the only identifiable feature being the cluster number. Any names or other identifiable features in the interview transcripts will be redacted. Only aggregated statistics and the results of analyses will be presented in any public output from the project. The data will be stored for the duration of the analyses on a password-protected computer.

There are a number of planned outputs. First, a number of articles for publication in peer-reviewed journals will be prepared. Second, a full report detailing the findings from the evaluation will be prepared. This will be made freely available online. Its intended audience is implementers of CHW programmes around the world. Third, the results from the evaluation will be presented to officials from the $\mathrm{MOH}$, Malawi. Finally, the results of the analyses will be presented at relevant public and global health conferences where possible.

This study will provide what we believe to be the first comparison between two 'doses' of the CHW programme and thus important evidence regarding the optimal approach to deployment of this increasingly critical cadre of healthcare workers. ${ }^{28}$ We propose a rigorous study design and the use of qualitative work to help explain the quantitative findings and enable the Household Model to be improved over time.

\section{Author affiliations \\ ${ }^{1}$ Abwenzi Pa Za Umoyo/Partners In Health, Neno, Malawi \\ ${ }^{2}$ Partners In Health Liberia, Harper, Liberia \\ ${ }^{3}$ University of Warwick, Coventry, UK \\ ${ }^{4}$ Partners In Health, Boston, Massachusetts, USA \\ ${ }^{5}$ Ministry of Health, Neno, Malawi}

Contributors EBW, ELD, BN, CK, RG, AJ and LN conceptualised the household model and tailored it to the setting, and led the implementation of the model in Neno District. SIW, RJL, CT, ELD, EBW, RM and LD developed the methodology to evaluate the model, with SIW developing the quantitative analysis plan. AM drafted the qualitative methods and analysis. ELD prepared the first draft of the protocol. $\mathrm{KC}$ adapted the protocol to the $B M J$ format. All authors reviewed and revised subsequent protocol drafts.

Funding This research received no specific grant from any funding agency in the public, commercial or not-for-profit sectors.

Competing interests None declared. 
Patient consent Not required.

Ethics approval Malawian National Health Science Research Committee (\#16/11/1694) in Lilongwe, Malawi; Partners Healthcare Human Research Committee (\#2017P000548/PHS) in Somerville, Massachusetts; and the Biomedical and Scientific Research Ethics Sub-Committee (REGO-2017-2060) at the University of Warwick in Coventry, UK.

Provenance and peer review Not commissioned; externally peer reviewed.

Open access This is an open access article distributed in accordance with the Creative Commons Attribution Non Commercial (CC BY-NC 4.0) license, which permits others to distribute, remix, adapt, build upon this work non-commercially, and license their derivative works on different terms, provided the original work is properly cited, appropriate credit is given, any changes made indicated, and the use is non-commercial. See:Ohttp://creativecommons.org/licenses/by-nc/4.0/.

\section{REFERENCES}

1. Jerome G, Ivers LC. Community health workers in health systems strengthening: a qualitative evaluation from rural Haiti. AIDS 2010;24(Suppl 1):S67-S72.

2. Rich ML, Miller AC, Niyigena P, et al. Excellent clinical outcomes and high retention in care among adults in a community-based HIV treatment program in rural Rwanda. J Acquir Immune Defic Syndr 2012;59:e35-e42.

3. McBain RK, Petersen E, Tophof N, et al. Impact and economic evaluation of a novel HIV service delivery model in rural Malawi. AIDS 2017;31:1999-2006.

4. Jhpiego Corporation. Developing and Strengthening Community Health Worker Programs at Scale [Internet]. 2013 http://www.mchip. net/sites/default/files/mchipfiles/CHW_ReferenceGuide_sm.pdf

5. Calcagno C, Lobatto ME, Robson PM, et al; Challenges Facing Successful Scaling Up of Effective Screening for Cardiovascular Disease by Community Health Workers in Mexico and South Africa: Policy Implications. 28, 2016.

6. Satti H, Motsamai S, Chetane P, et al. Comprehensive approach to improving maternal health and achieving MDG 5: report from the mountains of Lesotho. PLoS One 2012;7:e42700.

7. Biesma RG, Brugha R, Harmer A, et al. The effects of global health initiatives on country health systems: a review of the evidence from HIV/AIDS control. Health Policy Plan 2009;24:239-52.

8. Macinko J, Harris MJ. Brazil's family health strategy-delivering community-based primary care in a universal health system. $N$ Engl J Med 2015;372:2177-81.

9. Diaz T, Aboubaker S, Young M. Current scientific evidence for integrated community case management (iCCM) in Africa: Findings from the iCCM Evidence Symposium. J Glob Health 2014:4:20101.

10. Gilmore B, McAuliffe E. Effectiveness of community health workers delivering preventive interventions for maternal and child health in low- and middle-income countries: a systematic review. BMC Public Health 2013;13:847.

11. Smith S, Deveridge A, Berman J, et al. Task-shifting and prioritization: a situational analysis examining the role and experiences of community health workers in Malawi. Hum Resour Health 2014:12:12

12. Condo J, Mugeni C, Naughton B, et al. Rwanda's evolving community health worker system: a qualitative assessment of client and provider perspectives. Hum Resour Health 2014;12:1-7.

13. Kok MC, Ormel H, Broerse JEW, et al. Optimising the benefits of community health workers' unique position between communities and the health sector: A comparative analysis of factors shaping relationships in four countries. Glob Public Health 2017;12:1-29.

14. Malawi National Statistical Office. 2008 population and housing census: population projections. 2008 http://www.nsomalawi.mw/ images/stories/data_on_line/demography/census_2008/Main\% 20Report/Census\%20Main\%20Report.pdf

15. Malawi National Statistical Office. Malawi demographic and health survey. 2010 https://dhsprogram.com/pubs/pdf/FR247/FR247.pdf

16. The World Bank. World development indicators: GDP per capita. 2015 http://data. worldbank.org/indicator/NY.GDP.PCAP.CD

17. Malawi National AIDS Commission, Malawi Ministry of Health Department of HIV and AIDS, Joint United Nations Programme on HIV/AIDS. Press Release: 2014 National HIV and AIDS Estimates. 2015 http://www.aidsmalawi.org.mw/index.php/press-release? download=135: press-release-2014-hiv-estimates

18. Floyd K, Falzon D, Getahun $\mathrm{H}$, et al; Use of high burden country lists for TB by WHO in the post-2015 era, 2015.

19. Malawi National Statistical Office. MDG Endline Survey 2014. Zomba, Malawi. 2015 http://www.nsomalawi.mw/images/stories/ data_on_line/demography/MDG\%20Endline/MES\%202014\% 20Report.pdf

20. Malawi National Statistical Office. Malawi Demographic and Health Survey. 2016 https://dhsprogram.com/pubs/pdf/FR319/FR319.pdf

21. Malawi Ministry of Health, World Health Organization. Malawi 2009 National STEPS Survey for Chronic Non-Communicable Diseases and their Risk Factors Final Report. 2010 http://www.who.int/chp/ steps/Malawi 2009 STEPS Report.pdf

22. Hemming K, Lilford R, Girling AJ. Stepped-wedge cluster randomised controlled trials: a generic framework including parallel and multiple-level designs. Stat Med 2015;34:181-96.

23. Hemming K, Haines TP, Chilton PJ, et al. The stepped wedge cluster randomised trial: rationale, design, analysis, and reporting. BMJ 2015;350:h391.

24. Gordóvil-Merino A, Guàrdia-Olmos J, Peró-Cebollero M. Estimation of Logistic Regression Models in Small Samples. A Simulation Study Using a Weakly Informative Default Prior Distribution. Psicologica: International Journal of Methodology and Experimental Psychology 2012;33:345-61.

25. O'Hagan A, Stevens JW, Campbell MJ. Assurance in clinical trial design. Pharm Stat 2005;4:187-201.

26. O'Hagan A, Stevens JW. Bayesian assessment of sample size for clinical trials of cost-effectiveness. Med Decis Making 2001;21:219-30.

27. Spiegelhalter DJ. Bayesian methods for cluster randomized trials with continuous responses. Stat Med 2001;20:435-52.

28. World Health Organization. Health Workforce and Services. Draft Global Strategy on Human Resources for Health: Workforce 2030. Report by the Secretariat. WHO Executive Board, 2015. EB 138/36: 138th Session. Provisional Agenda Item 10.1. 